\title{
Epstein-Barr Virus-Induced Acute Hepatitis, Pancreatitis, and Pneumonitis in a Young Immunocompetent Adult: A Case Report
}

\author{
Authors: \\ Elias Fiani,, Rafca Challita, ${ }^{2}$ Hanaa Badawaki, ${ }^{3}$ Khaled Soukarieh, ${ }^{4}$ \\ Melissa Kyriakos Saad, ${ }^{5}$ *Elias Saikaly ${ }^{5}$ \\ 1. Department of Gastroenterology, Saint Georges Hospital University Medical Centre, \\ University of Balamand, Beirut, Lebanon \\ 2. Department of Rheumatology, Saint Georges Hospital University Medical Centre, \\ University of Balamand, Beirut, Lebanon \\ 3. Department of Nephrology, Geitawi Lebanese Hospital Medical Center, Lebanese \\ University, Beirut, Lebanon \\ 4. Internal Medicine Department, Al Zahraa Hospital University Medical Center, \\ Lebanese University, Beirut, Lebanon \\ 5. Department of General Surgery, Saint Georges Hospital University Medical Center, \\ University of Balamand, Beirut, Lebanon \\ *Correspondence to dreliassaikaly@gmail.com
}

Disclosure:

The authors have declared no conflicts of interest.

Received:

27.03.21

Accepted:

15.10 .21

Keywords:

Atypical pneumonia, Epstein-Barr virus (EBV), hepatitis, immunocompetent, pancreatitis, pneumonitis, respiratory failure.

Citation:

EMJ Respir. 2021; DOI/10.33590/emjrespir/21-00050.

\section{Abstract}

Epstein-Barr virus (EBV) is a common herpes virus (human herpesvirus type 4) that usually manifests as infectious mononucleosis or persists asymptomatically for life. EBV can also be associated with different types of malignancy such as T cell lymphoma, B cell lymphoma, Hodgkin lymphoma, and oropharyngeal squamous cell and nasopharyngeal carcinoma. Pneumonia is a very rare complication of EBV infection, but it has been reported to occur even in the absence of mononucleosis.

This article highlights the case of 35-year-old female who developed acute pancreatitis and acute respiratory failure related to EBV infection. The patient progressively recovered on antiviral therapy and steroids.

\section{INTRODUCTION}

Infectious mononucleosis (IM), caused by Epstein-Barr Virus (EBV), has been recognised as a clinical syndrome consisting of a triad of fever, tonsillar pharyngitis, and lymphadenopathy. Patients with IM can also present different skin manifestations or atypical exanthems, most commonly the pruritic maculopapular rash in patients receiving $\beta$-lactam antibiotics. ${ }^{2}$ Symptomatic infection with EBV is more likely to occur in adolescent and adult years, while primary EBV infections in children are often asymptomatic. $^{3}$ Some data suggest that older patients are more susceptible to develop a more severe clinical condition. ${ }^{4}$ In addition, elderly 
patients may also develop EBV-positive diffuse large B cell non-Hodgkin lymphoma, ${ }^{5}$ which has a poor prognosis and necessitates early diagnosis and treatment. ${ }^{6}$ EBV is also found to be associated not only with nasopharyngeal carcinoma, but also with oral and oropharyngeal squamous cell carcinomas. ${ }^{7}$ EBV can affect virtually any organ system and has been associated with different disease manifestations such as hepatitis or cholestasis, jaundice, hepatomegaly, pneumonia, pleural effusions, and pancreatitis..$^{8-10}$ Herein, the authors present a case of an immunocompetent adult who developed respiratory failure due to EBV pneumonitis along with cholestatic hepatitis and pancreatitis, without the suggestive clinical manifestations of IM.

\section{CASE REPORT}

A 35-year-old female with no significant past medical history presented to the emergency department with a complaint of epigastric pain which was non-radiating, had increased in intensity over time, and was associated with a fever of three days duration. The patient denied prior abdominal surgeries or trauma. They also denied any travel history, alcohol intake, or recreational drug use. The patient noted no inherited medical conditions in their family.

On physical examination, the patient was tachycardic (105 beats per $\mathrm{min}$ ) and febrile (temperature of $38.5^{\circ} \mathrm{C}$ ), and their pulse oximeter saturation was measured at $98 \%$. Diffuse jaundice was noted. There was no palpable cervical or supraclavicular lymphadenopathy, and no tonsillar exudates. On abdominal palpation, they had severe right upper quadrant and epigastric tenderness, but no hepatomegaly or splenomegaly.

Laboratory investigations revealed the following results: white blood cell count: 10,440 /L; neutrophils: 70\%; haemoglobin: 13.8 g/dL; haematocrit: 40\%; mean corpuscular volume: $94 \mathrm{fL}$; platelet count: $300,000 / \mathrm{mm}^{3}$; creatinine: $0.7 \mathrm{mg} / \mathrm{dL}$; aspartate transmaninase: $448 \mathrm{IU} / \mathrm{L}$; alanine aminotransferase: $110 \mathrm{IU} / \mathrm{L}$; alkaline phosphatase: $150 \mathrm{IU} / \mathrm{L} ; \mathrm{V}$-glutamyltransferase: 1,670 IU/L; lipase: $408 \mathrm{IU} / \mathrm{L}$; amylase: $129 \mathrm{U} / \mathrm{L}$; C-reactive protein: $221 \mathrm{mg} / \mathrm{L}$; triglycerides: $150 \mathrm{mg} / \mathrm{dL}$; international normalised ratio: 1.1; albumin: $3.5 \mathrm{~g} / \mathrm{dL}$; and total bilirubin of $15.9 \mathrm{mg} / \mathrm{dL}$, with direct bilirubin of $12 \mathrm{mg} / \mathrm{dL}$.
Abdominal ultrasonography showed no evidence of acute cholecystitis, normal calibre of the common bile duct, and no gallstones. A CT scan of the abdomen and pelvis showed diffuse increase in the volume of the pancreas with no sign of necrosis, along with peripancreatic inflammatory changes. No definite abscess or collection was seen on the CT scan. An abdominal MRI with gadolinium and magnetic resonance cholangiopancreatography revealed non-complicated pancreatitis, with absence of intra- or extra-biliary ductal dilatation or common bile duct stones.

Results of serologic tests for hepatitis A, B, and C were as follows: hepatitis B surface antigen ( $\mathrm{Ag}$ ) test: 0.16 (negative); hepatitis B core antibody (Ab) IgM: non-reactive; hepatitis B Ab total: non-reactive; hepatitis $C$ virus Ab: 0.13 (negative); and hepatitis A virus IgM Ab: 0.12 (negative). HIV (1+2) Ag/Ab was negative. Abs for cytomegalovirus (IgM: 0.7); mycoplasma pneumonia Abs (IgM <7.7); antinuclear Abs (<1:100); anti-mitochondrial Abs (<1:100); and anti-phospholipids Abs (anti-cardiolipin Abs <2, anti- $\beta-2$ glycoprotein Abs: 9.4) were all negative. Work-up for haemochromatosis and Wilson's disease were also negative. EBV viral load in serum was 625 copies /mL; EBV nuclear Ag IgG was negative; IgM for EBV viral capsid Ag (10.24) and IgG for EBV viral capsid Ag (44.08) were positive, confirming acute primary EBV infection.

The patient was diagnosed with acute pancreatitis associated with acute cholestatic hepatitis, induced by a primary EBV infection. Bowel rest and intravenous (IV) hydration were instituted. Over the next two days, high-grade fever persisted, and the patient developed acute respiratory failure with need for mechanical ventilation (fraction of inspired oxygen: 100\%; positive end-expiratory pressures: 14). Chest X-ray and CT scans (Figures 1-3) showed bilateral pleural effusion with diffuse ground glass appearance and left consolidation. The echocardiography was unremarkable.

As a bacterial co-infection could not be ruled out based on the chest CT scan findings, linezolid and meropenem were administered, in addition to antiviral drugs (oseltamivir and acyclovir), and methylprednisolone (at a dose of 40 mg IV every 12 hours at $1 \mathrm{mg} / \mathrm{kg}$ ). PCR of EBV in deep tracheal aspirates was positive with a high viral load of $24,860,000$ copies/mL, confirming the diagnosis 


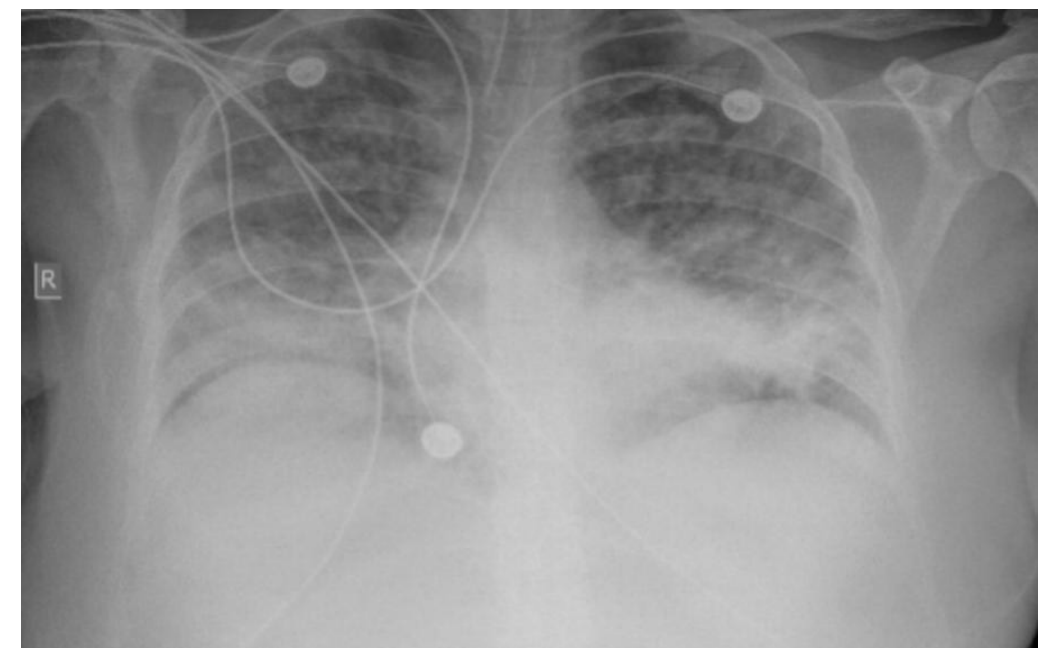

Figure 1: Chest X-ray showing bilateral infiltrates.

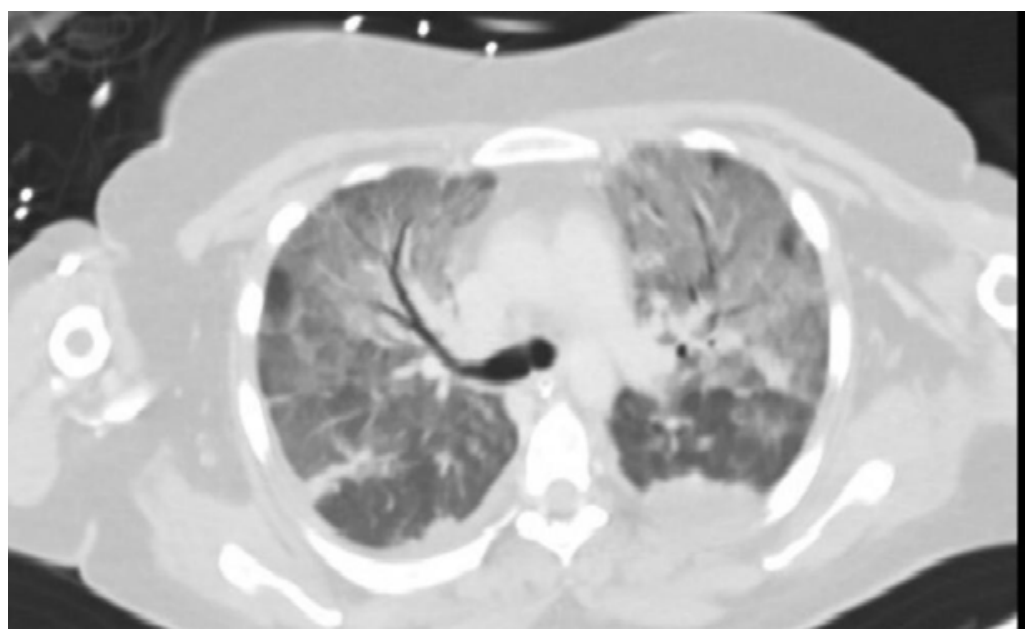

Figure 2: CT chest showing bilateral ground glass appearance.

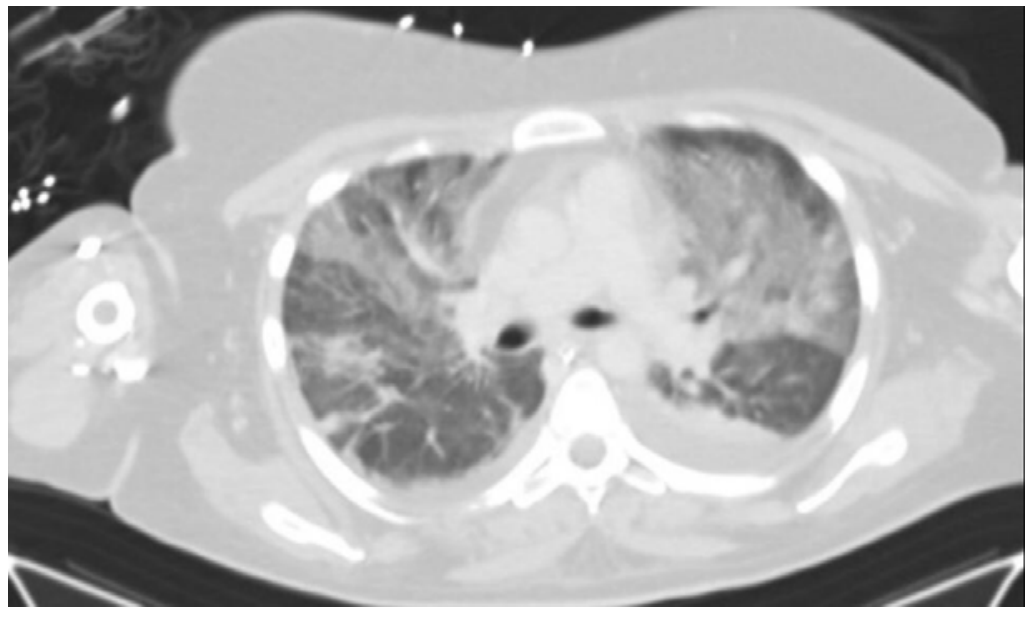

Figure 3: CT chest showing left consolidation. 
of EBV pneumonitis with type one respiratory failure, in addition to acute pancreatitis and cholestatic hepatitis. The patient received acyclovir for 2 weeks and IV steroids for 1 week, which was stopped following the improvement of her condition. Jaundice spontaneously resolved over the next few days. Liver function tests and liver enzymes progressively returned to a normal level, and serum lipase levels steadily decreased. The patient was extubated on Day 10 and discharged on Day 14. EBV serum viral load and EBV serology were ordered, but unfortunately could not be carried out due to financial reasons. The patient has remained well, with follow-up visits occurring over a period of 7 months.

\section{DISCUSSION}

This article presents the case of a young female whose main complaints were abdominal pain, jaundice, and fever, with no clear symptoms of infectious mononucleosis such as pharyngitis and lymphadenopathy. Based on the laboratory and imaging findings, the first diagnosis was acute primary EBV infection with acute pancreatitis and cholestatic hepatitis. The clinical deterioration of the patient into respiratory failure warranted further investigations, including EBV PCR from endotracheal aspirate. The extensive work-up led to an additional diagnosis of EBV pneumonitis, although the first chest X-ray on admission was unremarkable. After confirmation of the diagnosis, the treatment of EBV pneumonia consisted mainly of IV acyclovir and steroids. The patient had remarkable clinical improvement, along with radiological resolution of pneumonitis, alongside recovery from pancreatitis and hepatitis with conservative measures.

The majority of individuals remain asymptomatic during EBV infection, or may develop IM, which is relatively common and self-limited. Although it is infrequent, EBV infection can lead to severe and serious complications." To the best of the authors' knowledge, five cases of EBV infection associated with acute pancreatitis and hepatitis have been reported in the literature..$^{12,13}$ Unexplained abdominal pain in patients with EBV infection should raise suspicion of acute pancreatitis. ${ }^{14}$ This patient had fulfilled the three diagnostic criteria of acute pancreatitis based on the American College of Gastroenterology (ACG) guidelines: abdominal pain, elevated serum lipase and/or amylase level (greater than three times the upper normal limit), and radiological evidence of acute pancreatitis. ${ }^{15}$ In addition, the presence of fever and disturbed liver function tests, in the absence of cholelithiasis on imaging, can occur in the setting of EBV-induced cholestatic hepatitis. ${ }^{10}$ Hepatitis and pancreatitis associated with EBV infection have been managed, as mentioned in the literature, mainly with symptomatic and supportive treatment, which was associated with good results overall. ${ }^{16}$

Apart from gastrointestinal involvement, lung involvement is infrequently associated with EBV infection; it is more commonly encountered in immunosuppressed patients, but can be reported in healthy subjects as well. ${ }^{1718}$ Pulmonary manifestations in EBV can involve lymphadenopathy (mainly hilar and mediastinal lymph nodes), pleural effusions, and interstitial pneumonitis. ${ }^{19}$ The mechanism of pulmonary involvement in EBV infection remains controversial; it is unclear whether it results from direct viral invasion and replication in lung tissue, immunological reaction to the infection, or a combination of both. ${ }^{20}$ Although no specific antiviral drug has precedence over others, favourable outcomes were reported with the use of IV acyclovir in patients with EBV pneumonitis. ${ }^{21,22}$ Timely diagnosis is pivotal in treating severe EBV infections. In fact, early clinical and radiological recoveries were achieved in patients with severe EBV pulmonary infection by initiating antiviral therapy early in the course of the disease. ${ }^{23}$ As for the cases of delayed diagnosis, corticosteroids, Ig, and IV antivirals were used together and yielded successful results. ${ }^{23}$ Recovery from EBV pneumonitis by receiving steroid medication alone without antiviral agents was also reported. ${ }^{19}$

\section{CONCLUSION}

Lung involvement, hepatitis, and cholestasis following EBV infection are considered rare manifestations of the infection, with a high risk of mortality. This article presented an uncommon case of EBV-induced acute cholestatic hepatitis, pancreatitis, and pneumonitis in an immunocompetent patient. The clinical presentation was severe, and the patient needed intubation. They were successfully treated with IV acyclovir and steroids, which resulted in a satisfactory outcome. 


\section{References}

1. Fugl A, Andersen CL. EpsteinBarr virus and its association with disease - a review of relevance to general practice. BMC Fam Pract. 2019;20(1):62.

2. Ciccarese $\mathrm{G}$ et al. Dermatological manifestations of Epstein-Barr virus systemic infection: a case report and literature review. Int J Dermatol. 2020;59(10):1202-9.

3. Dunmire SK et al. Infectious mononucleosis. Curr Top Microbiol Immunol. 2015;390(1):211-40.

4. Hocqueloux $L$ et al. The high burden of hospitalizations for primary EBV infection: a 6-year prospective survey in a French hospital. Clin Microbiol Infect. 2015;21(11):1041.

5. Smeltzer J et al. Epstein-Barr virus infection in an elderly nonimmunocompromised adult successfully treated with rituximab. Case Rep Hematol. 2014;2014:641483.

6. Murthy SL et al. Epstein-Barr virus-positive diffuse large B-cell lymphoma. Proc (Bayl Univ Med Cent). 2017:30(4):443-4.

7. Broccolo F et al. Human papillomavirus (HPV) and EpsteinBarr virus (EBV) in kerat-inizing versus non- keratinizing squamous cell carcinoma of the oropharynx. Infect Agent Cancer. 2018;13:32.

8. Nowalk A, Green M. Epstein-Barr virus. Microbiol Spectr. 2016;4(3).

9. Khoo A et al. Acute cholestatic hepatitis induced by Epstein-Barr virus infection in an adult: a case report. J Med Case Rep. 2016;10:75.

10. Chen $\mathrm{J}$ et al. Just another simple case of infectious mononucleosis? Lancet. 2003;361(9364):1182.

11. Vouloumanou EK et al. Current diagnosis and management of infectious mononu-cleosis. Curr Opin Hematol. 2012;19(1):14-20.

12. Khawcharoenporn $T$ et al. EpsteinBarr virus infection with acute pancreatitis. Int J Infect Dis. 2008;12(2):227-9.

13. Wislocki LC. Acute pancreatitis in infectious mononucleosis. N Engl J Med. 1966;275(6):322-3.

14. Hammami MB et al. Epstein-Barr virus-associated acute pancreatitis. BMJ Case Rep. 2019;14;12(11):e231744

15. Tenner $\mathrm{S}$ et al. American College of gastroenterology guideline: management of acute pancreatitis. Am J Gastroenterol. 2013;108(9):1400-15.

16. Kang SJ et al. Epstein-barr virus infection with acute pancreatitis associated with cholestatic hepatitis. Pediatr Gastroenterol Hepatol Nutr. 2013:16(1):61-4.

17. Odumade OA et al. Progress and problems in understanding and managing primary Epstein-Barr virus infections. Clin Microbiol Rev. 2011;24(1):193-209.

18. Nieweld $\mathrm{C}$ et al. Pneumonitis secondary to EBV-specific cytotoxic T- Iymphocytes. Chest. 2018;154(Suppl 4):423A. DOI: 10.1016/j.chest.2018.08.386

19. Niazi MR et al. Epstein-Barr virus (EBV) induced pneumonitis in an immunocompe-tent adult: a case report. Respir Med Case Rep. 2020;31:101262.

20. Krumbholz A et al. Epstein-Barr virus-associated pneumonia and bronchiolitis oblit-erans syndrome in a lung transplant recipient. Med Microbiol Immunol. 2010;199(4):317 22.

21. Kimura H. Pathogenesis of chronic active Epstein-Barr virus infection: is this an in-fectious disease, lymphoproliferative disorder, or immunodeficiency? Rev Med Vi-rol. 2006;16(4):251-61.

22. Terence E et al. Epstein-Barr virus pneumonitis. Ulster Med J. 2009;78(2):137-8.

23. Yin $Q$ et al. Immunocompetent adult infected with Epstein-Barr virus presenting as severe respiratory insufficiency: a case report. Int J Clin Exp Med. 2017;10(2):4011-3. 